\title{
Accretion disk wind in the AGN broad-line region: Spectroscopically resolved line profile variations in Mrk 110
}

\author{
W. Kollatschny $\star \star$
}

\author{
Universitäts-Sternwarte Göttingen, Geismarlandstraße 11, 37083 Göttingen, Germany \\ Department of Astronomy and McDonald Observatory, University of Texas at Austin, Austin, TX 78712, USA
}

Received 1 April 2003 / Accepted 12 June 2003

\begin{abstract}
Detailed line profile variability studies of the narrow line Seyfert 1 galaxy Mrk 110 are presented. We obtained the spectra in a variability campaign carried out with the $9.2 \mathrm{~m}$ Hobby-Eberly Telescope at McDonald Observatory. The integrated Balmer and helium (He I, II) emission lines are delayed by 3 to 33 light days to the optical continuum variations respectively. The outer wings of the line profiles respond much faster to continuum variations than the central regions. The comparison of the observed profile variations with model calculations of different velocity fields indicates an accretion disk structure of the broad line emitting region in Mrk 110. Comparing the velocity-delay maps of the different emission lines among each other a clear radial stratification in the BLR can be recognized. Furthermore, delays of the red line wings are slightly shorter than those of the blue wings. This indicates an accretion disk wind in the BLR of Mrk 110. We determine a central black hole mass of $M=1.8 \times 10^{7} M_{\odot}$. Because of the poorly known inclination angle of the accretion disk this is a lower limit only.
\end{abstract}

Key words. accretion, accretion disks - line: profiles - galaxies: Seyfert - galaxies: individual: Mrk 110 - galaxies: nuclei galaxies: quasars: emission lines

\section{Introduction}

The central broad emission line region (BLR) in active galactic nuclei is unresolved. But a study of the variable ionizing continuum source and the delayed response of the broad emission lines provides indirect information about size and structure of the line emitting region and their internal kinematics. In this paper we present a detailed study of continuum and emission line profile variations in the Seyfert 1 galaxy Mrk 110. Our goal is to determine the structure and kinematics of its broad-line region on the one hand and its central black hole mass on the other hand.

The study of the kinematics in the central broad-line region of AGN requires the acquisition of high $S / N$ spectra for analyzing in detail velocity resolved line profiles. Furthermore, a homogeneous set of spectra has to be obtained over months with spacings of days to weeks. A comparison of the evolution of line profiles with theoretical models (e.g. Welsh \& Horne 1991) can give us information on the kinematics in the broadline region i.e. whether radial inflow or outflow motions, turbulent/chaotic velocity fields, or Keplerian orbits are dominant.

We published first results of a variability campaign of Mrk 110 with the $9.2 \mathrm{~m}$ Hobby-Eberly-Telescope in

* Based on observations obtained with the Hobby-Eberly Telescope, which is a joint project of the University of Texas at Austin, the Pennsylvania State University, Stanford University, Ludwig-Maximilians-Universität München, and Georg-AugustUniversität Göttingen.

$\star \star$ e-mail: wkollat@uni-sw.gwdg.de
Kollatschny et al. 2001 (hereafter called Paper I). We verified the extent and stratification of the BLR. Results on the velocity field based on $\mathrm{H} \beta$ variations have been published in Kollatschny \& Bischoff (2002, hereafter called Paper II) indicating that the broad-line region is connected with a central accretion disk.

Theoretical models of accretion disk outflow scenarios in AGN have been published by different authors. They investigated radiatively-driven wind models (e.g. Murray \& Chiang 1997; Proga et al. 2000) and/or magnetically-driven disk outflow models (Blandford \& Payne 1982; Bottorff et al. 1997; Emmering et al. 1992; Königl \& Kartje 1994).

There are other pieces of evidence from multi-frequency and/or spectro-polarimetric observations in the literature that the BLR is connected with an accretion disk (Elvis 2000; Cohen \& Martel 2002).

In this paper we present new velocity delay maps of several Balmer and helium emission lines in the spectrum of Mrk 110 to verify the accretion disk scenario in the BLR of this galaxy.

\section{Observations and data reduction}

The observations of this monitoring campaign were carried out at the $9.2 \mathrm{~m}$ Hobby-Eberly Telescope (HET) at McDonald Observatory. We took 26 spectra between 1999 November 13 (JD 2451495 ) and 2000 May 14 (JD 2451678 ) with a median interval of 3 days. A log of the observations is presented in Paper I. 
We obtained all our spectra under identical conditions with the Low Resolution Spectrograph (LRS) located at the prime focus of the HET. A Ford Aerospace CCD $(3072 \times 1024)$ with $15 \mu \mathrm{m}$ pixel was used throughout our monitoring run. The spectra cover a wavelength range from $4200 \AA$ to $6900 \AA$ with a resolving power of 650 at $5000 \AA$. Exposure times were 10 to $20 \mathrm{~min}$ and yielded a $S / N>100$ per pixel in the continuum in most cases.

We reduced the spectra in a homogeneous way with IRAF reduction packages including bias subtraction, cosmic ray correction, flat-field correction, wavelength calibration, night sky subtraction and flux calibration. Great care was taken to obtain very good intensity and wavelength calibration by using the spatially unresolved forbidden emission lines in the spectra as additional internal calibrators.

We generated first a mean spectrum of our variability campaign with very high $S / N$. Afterwards we calculated difference spectra of all epochs with respect to this mean spectrum. We adapted our spectra by minimizing the residuals of the constant narrow emission lines in the difference spectra. The primary lines for the internal calibration were the [OIII] $\lambda 5007, \lambda 4959$ lines. Thus we corrected for small spectral shifts, for minor differences in the spectral resolution caused by different seeing conditions and for small scaling factors. We used the narrow components of the Balmer and helium lines as well as the [OI] $26300, \lambda 6363$, [SII] $\lambda 6717, \lambda 6731$, and [NII] $\lambda 6548, \lambda 6584$ emission lines as secondary calibrators to secure the calibration over the whole spectral range. In this way we achieved relative line fluxes with an accuracy of better than $1 \%$ in most of the spectra. The main error sources were the variable broad HeI $\lambda 5016, \lambda 4922$ emission lines blending the $[\mathrm{OIII}] \lambda 5007, \lambda 4959$ calibration lines. Different seeing conditions during the observations caused slightly different stellar contributions from the underlying host galaxy in our spectra. This effected the pseudo-continuum we used to subtract from the spectra. Further sources of error are the slopes of the sensitivity functions we derived from our standard star spectra. At the edges of the spectrum one can get varieties of $1 \%$ to $3 \%$ in the sensitivity function by fitting the observed bandpasses. This impreciseness influenced especially the $\mathrm{H} \alpha$ and $\mathrm{H} \gamma$ line profiles. Furthermore, the $\mathrm{H} \gamma$ line is heavily blended with the [OIII] $\lambda 4363$ line and the $\mathrm{H} \alpha$ line is heavily contaminated by the variable atmospheric absorption. The errors in the intensities of these strong Balmer line are therefore comparable to those of the weaker helium lines.

Further details of the observations and reduction procedure are published in Papers I and II.

\section{Results}

We concentrated on integrated line intensity variations of the broad emission lines as well as on line profile variations in the $\mathrm{H} \beta$ line in our Papers I and II. Here we present results on line profile variations in several Balmer and helium lines. These line profiles must have a large $S / N$ ratio for doing this study.

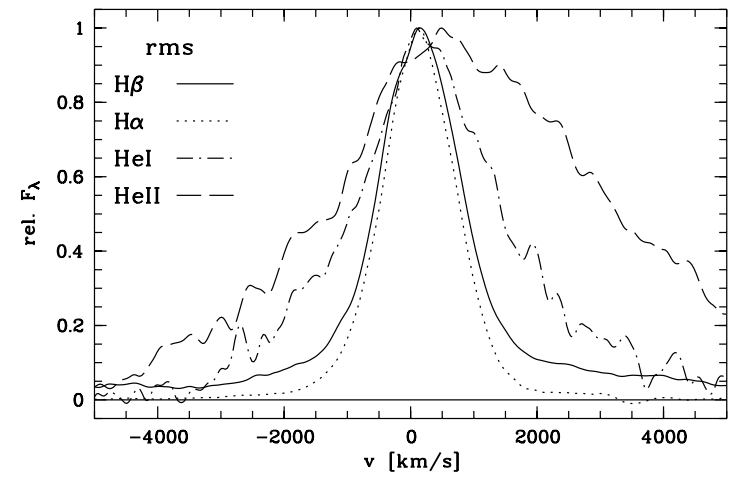

Fig. 1. Normalized Balmer and helium rms line profiles in velocity space.

Table 1. Rms line widths ( $F W H M)$ of the strongest emission lines, their cross-correlation lags with respect to continuum variations, and the derived central black hole masses (see text for details).

\begin{tabular}{lccc}
\hline \hline Line & $\begin{array}{c}F W H M(\mathrm{rms}) \\
{\left[\mathrm{km} \mathrm{s}^{-1}\right]} \\
(2)\end{array}$ & $\begin{array}{c}\tau_{\text {cent }} \\
{[\text { days }]} \\
(3)\end{array}$ & $\begin{array}{c}M \\
{\left[10^{7} M_{\odot}\right]}\end{array}$ \\
\hline HeII $\lambda 4686$ & $4444 . \pm 200$ & $3.5_{-2 .}^{+2 .}$ & $2.25_{-0.45}^{+1.63}$ \\
HeI $\lambda 5876$ & $2404 . \pm 100$ & $10.8_{-4 .}^{+4 .}$ & $1.81_{-0.33}^{+1.36}$ \\
H $\beta$ & $1515 . \pm 100$ & $23.5_{-4 .}^{+4 .}$ & $1.63_{-0.31}^{+0.33}$ \\
$\mathrm{H} \alpha$ & $1315 . \pm 100$ & $32.5_{-4 .}^{+4 .}$ & $1.64_{-0.35}^{+0.33}$ \\
\hline
\end{tabular}

\subsection{Integrated line intensity variations and rms line profiles}

We derived mean and rms line profiles from all the spectra obtained at our HET variability campaign. The rms spectra give us the variable part of the emission lines profiles we are investigating here. The constant narrow lines in the galaxy spectra cancel out in the rms spectra. The normalized rms line profiles of the strongest Balmer $(\mathrm{H} \alpha, \mathrm{H} \beta)$ and helium emission lines (HeII $\lambda 4686, \mathrm{HeI} \lambda 5876$ ) are presented in Fig. 1 in velocity space. We did not consider the $\mathrm{H} \gamma$ line in this figure because its rms line profile is still contaminated by the strong [OIII] $\lambda 4363$ line blend. The big differences in the widths of these lines are obvious. The helium lines and especially the HeII line are much broader than the Balmer lines. We published in Paper I (Fig. 7) the entire rms spectrum of Mrk 110. One can see that structures in the broad He line wings are real and not caused by noise by comparing them with the $S / N$ ratio in the continuum. The measured rms line widths $(F W H M)$ of these profiles are listed in Table 1.

In Paper I we derived the mean distance $\tau_{\text {cent }}$ of these line emitting regions from the central ionizing source. This was done by calculating the cross-correlation function of the integrated line light curves with the variable ionizing continuum light curve. These results are given in Table 1 as well for completeness. In Fig. 2 we plot the derived time lags of the Balmer and helium emission lines as a function of their FWHM of the rms line profiles. These time lags can be interpreted as the lighttravel time across the emission region for the different broad 


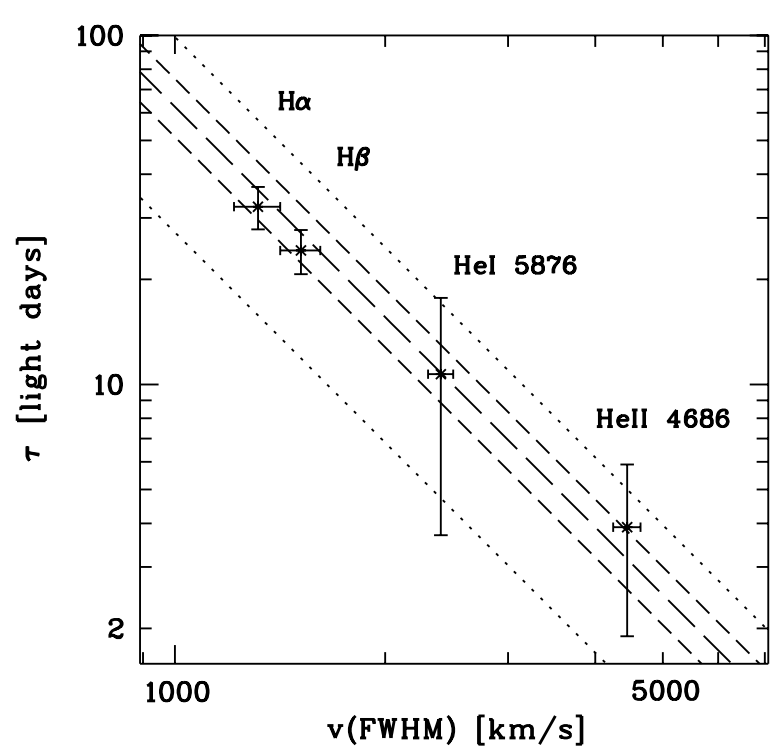

Fig. 2. The distance of the Balmer and helium emitting line regions from the central ionizing source in Mrk 110 as a function of the FWHM in their rms line profiles. The dotted and dashed lines are the results from our model calculations for central masses of $0.8,1.5,1.8$, 2.2, and $2.9 \times 10^{7} M_{\odot}$ (from bottom to top).

emission lines. There is a clear correlation between line width and time lag.

We interprete the light-travel time as the characteristic distance $R$ of the line emitting region and the FWHM of the rms emission line width as the characteristic velocity $v$ of the line emitting clouds. We estimate the central masses of the central black hole under the assumption that the gas dynamics are dominated by a central massive object (see Paper I). Additionally, we calculated the relation between radius and velocity for different black hole masses. The dotted and dashed lines shown in Fig. 2 correspond to virial masses of 0.8, 1.5, $1.8,2.2$, and $2.9 \times 10^{7} M_{\odot}$ (from bottom to top). It is evident that the black hole mass of $1.8 \times 10^{7} M_{\odot}$ we derived in Paper I from the observed data of the individual emission lines matches the calculated model relation. But we have to keep in mind that there might be additional systematic uncertainties in the determination of the central black hole mass due to not considered geometry and/or orientation effects of the BLR in these models.

\subsection{Light curves of emission line segments}

The light curves of the continuum flux at $5135 \AA$ and of the integrated broad emission line intensities of our HET variability campaign are published in Paper I. The strongest broad emission lines in our spectra are the $\mathrm{H} \alpha, \mathrm{H} \beta$, and $\mathrm{H} \gamma$ Balmer lines and the HeII $\lambda 4686$ and HeI $\lambda 5876$ helium lines.

In this paper we concentrate on variations in the emission line profile only. First results of the $\mathrm{H} \beta$ profile variations have been published in Paper II. We measured the light curves of all subsequent velocity segments $\left(\Delta v=400 \mathrm{~km} \mathrm{~s}^{-1}\right.$ width) from $v=-5000$ until $+5000 \mathrm{~km} \mathrm{~s}^{-1}$ in the strongest Balmer and helium lines. The intensity of the central line segment is integrated from $v=-200$ until $+200 \mathrm{~km} \mathrm{~s}^{-1}$. The light curves of the line center as well as of blue and red line wing segments at $v=\mp 600,1200,2000 \mathrm{~km} \mathrm{~s}^{-1}\left(\Delta v=400 \mathrm{~km} \mathrm{~s}^{-1}\right)$ for HeII $\lambda 4686, \mathrm{HeI} \lambda 5876, \mathrm{H} \gamma$, and $\mathrm{H} \alpha$ are shown in Figs. 3 to 6 in addition to the continuum light curve. In Paper II we published corresponding light curves for $\mathrm{H} \beta$. In that case we used segments with a velocity binning of $\Delta v=200 \mathrm{~km} \mathrm{~s}^{-1}$. But these light curves are nearly identical to those resulting with a $\Delta v=400 \mathrm{~km} \mathrm{~s}^{-1}$ binning.

The $\mathrm{H} \gamma$ line segment light curves are heavily contaminated by other lines. The red wing is heavily blended with the strong [OIII] $\lambda 4363$ line (Paper I, Fig. 1) and the blue wing by FeII multiplets. The red wing of the redshifted $\mathrm{H} \alpha$ line is heavily contaminated by atmospheric absorption in addition to the blending by the [NII] $\lambda 6548,6584$ lines within the line profile.

The light curves of the segments in each line profile are remarkably different. There is the general trend that the pattern of the light curves varies as a function of distance to line center. On the other hand, corresponding light curves of identical red and blue segments are very similar. One can see immediately (Figs. 3-6) that the outer line wings follow closer the continuum light curve than the inner line wings. The errors given in the light curves indicate absolute errors. Relative errors between light curves of the different segments are smaller by about $50 \%$.

\subsection{Velocity delay maps of the Balmer and helium lines}

We computed cross-correlation functions (CCF) of all line segment $\left(\Delta v=400 \mathrm{~km} \mathrm{~s}^{-1}\right)$ light curves with the $5100 \AA$ continuum light curve. For details of the method see Papers I and II.

The derived delays of the segments are shown in Fig. 7 as function of distance to the line center. These velocity delay maps are presented in gray scale for the $\mathrm{H} \beta$, $\mathrm{HeI} \lambda 5876$, HeII $\lambda 4686, \mathrm{H} \alpha$, and $\mathrm{H} \gamma$ lines. The solid lines show contour lines of the correlation coefficient at levels between 0.800 and 0.925 .

The $\mathrm{H} \gamma$ line and the redshifted $\mathrm{H} \alpha$ line are heavily contaminated by other emission and/or absorption lines as had been said before. Therefore, the correlation coefficient is smaller in the wings of these lines. From now on we will consider only the $\mathrm{H} \beta, \mathrm{HeI} \lambda 5876$, and HeII $\lambda 4686$ lines for a more detailed discussion.

The light curves of the line center are mostly delayed with respect to continuum variations. The outer line wings respond much faster to continuum variations respectively than the inner line profile segments. Comparing the 2-D CCFs of the different lines with each other one can identify a clear stratification within the broad line region. The same trend has been seen before in the integrated lines. The HeII $\lambda 4686$ line responds first to continuum variations followed by $\mathrm{HeL} \lambda 5876$ and finally $\mathrm{H} \beta$. The correlation coefficients in the line wings as well as in the line centers of the $\mathrm{H} \beta$ and $\mathrm{He}$ lines are very similar although the intensities in the line wings are obviously smaller. But one has to keep in mind that the line wings originate closer to the nuclear ionizing source than the line centers. 

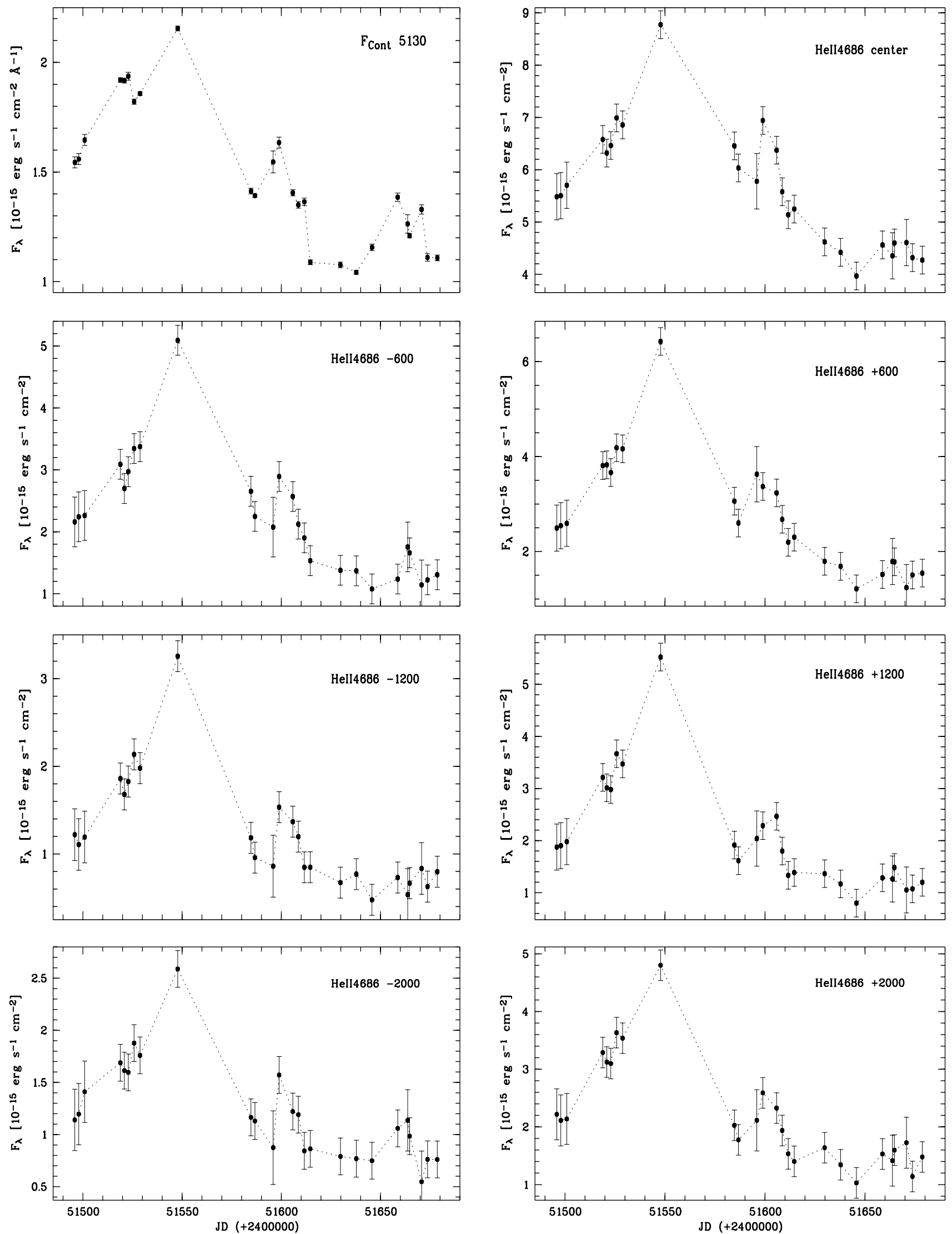

Fig. 3. Light curves of the continuum, of the HeII $\lambda 4686$ line center as well as of different blue and red HeII $\lambda 4686$ line wing segments $(v=\mp 600$, $1200,2000 \mathrm{~km} \mathrm{~s}^{-1}, \Delta v=400 \mathrm{~km} \mathrm{~s}^{-1}$ ) derived from our HET variability campaign of Mrk 110. 

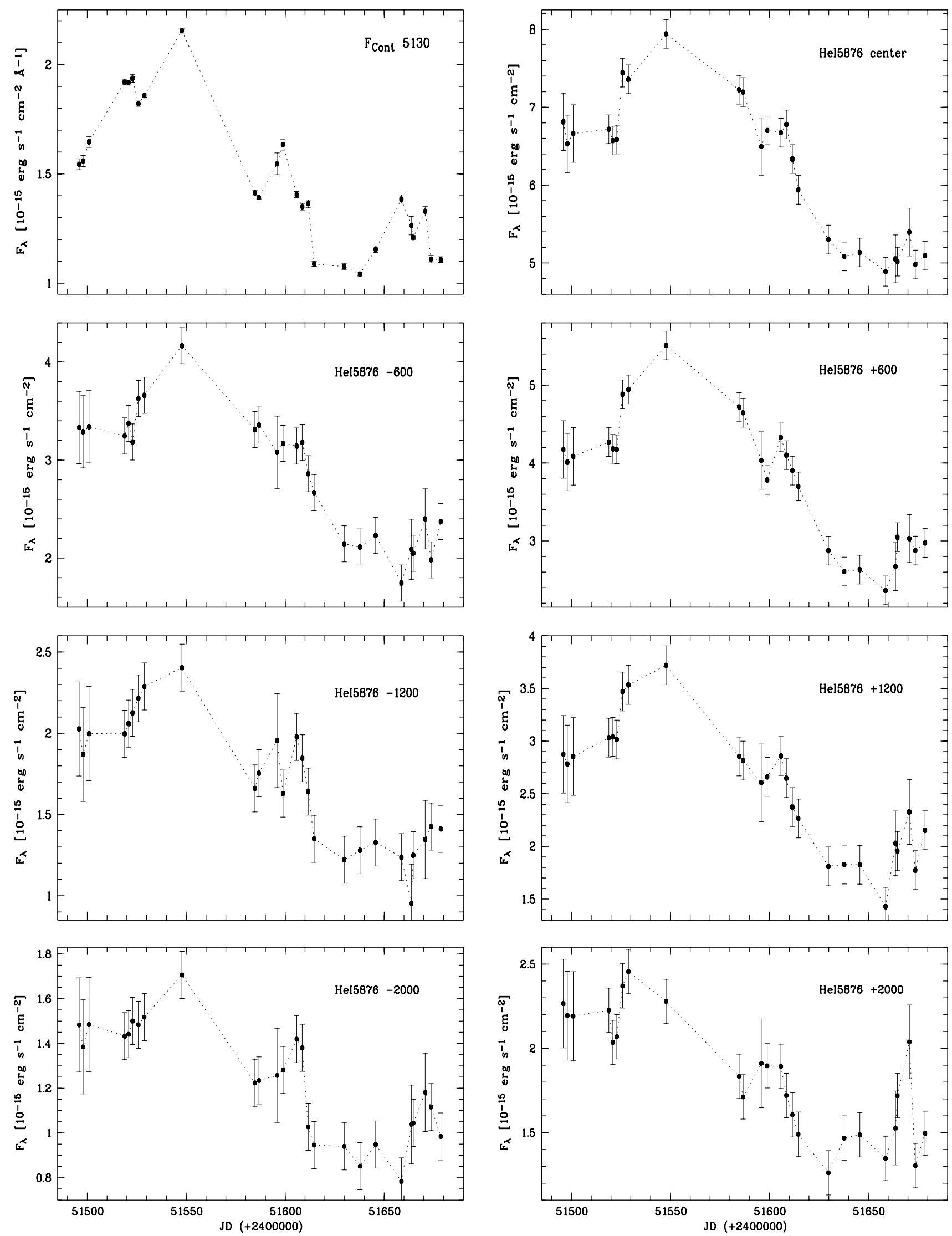

Fig. 4. Light curves of the continuum, of the HeI $\lambda 5876$ line center as well as of different blue and red HeI $\lambda 5876$ line wing segments $(v=\mp 600$, $1200,2000 \mathrm{~km} \mathrm{~s}^{-1}, \Delta v=400 \mathrm{~km} \mathrm{~s}^{-1}$ ) of Mrk 110. 

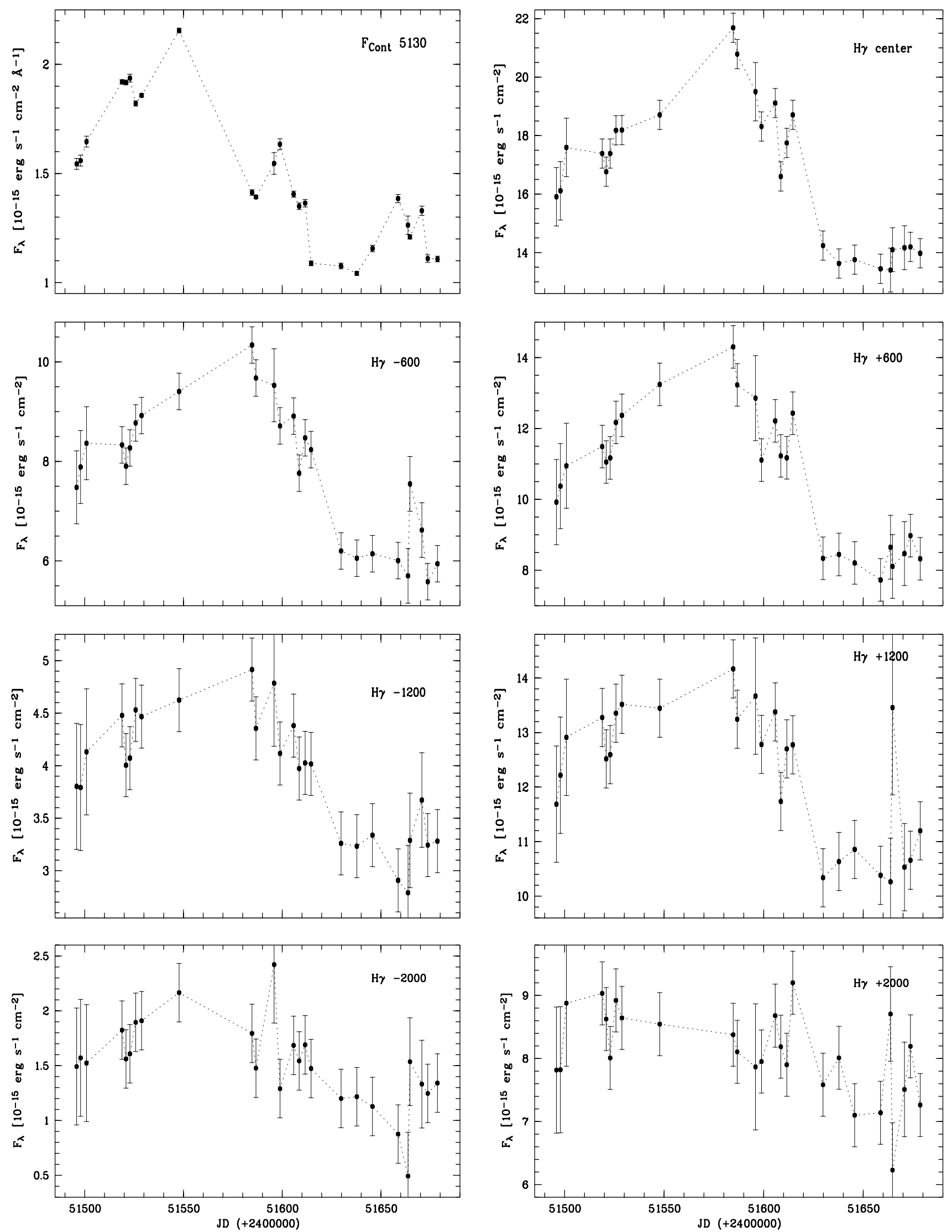

Fig. 5. Light curves of the continuum, of the $\mathrm{H} \gamma$ line center as well as of different blue and red $\mathrm{H} \gamma$ line wing segments $(v= \pm 600$, 1200, $2000 \mathrm{~km} \mathrm{~s}^{-1}, \Delta v=400 \mathrm{~km} \mathrm{~s}^{-1}$ ) of Mrk 110 . 

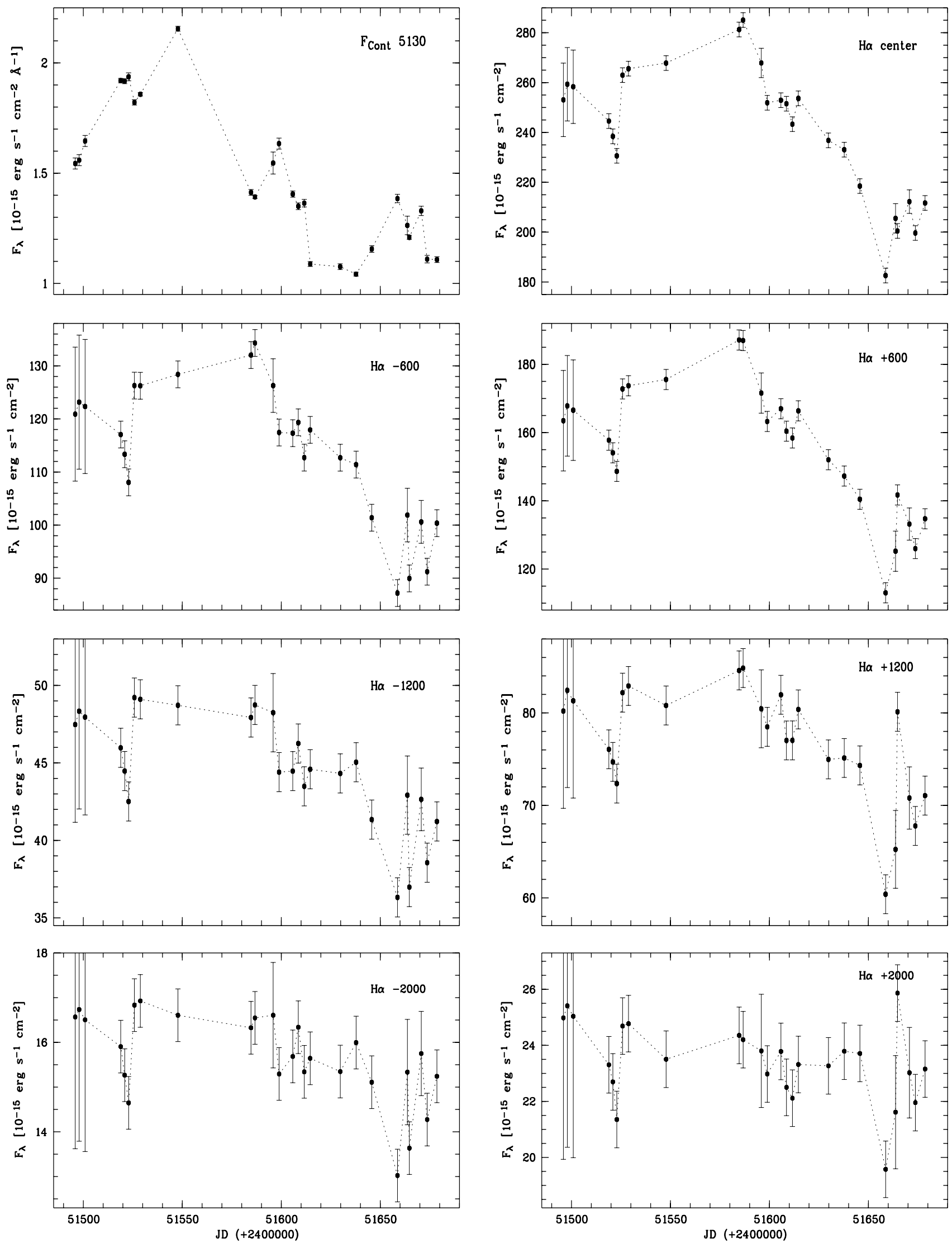

Fig. 6. Light curves of the continuum, of the $\mathrm{H} \alpha$ line center as well as of different blue and red $\mathrm{H} \alpha$ line wing segments $(v= \pm 600,1200$, $2000 \mathrm{~km} \mathrm{~s}^{-1}, \Delta v=400 \mathrm{~km} \mathrm{~s}^{-1}$ ) of Mrk 110 . 

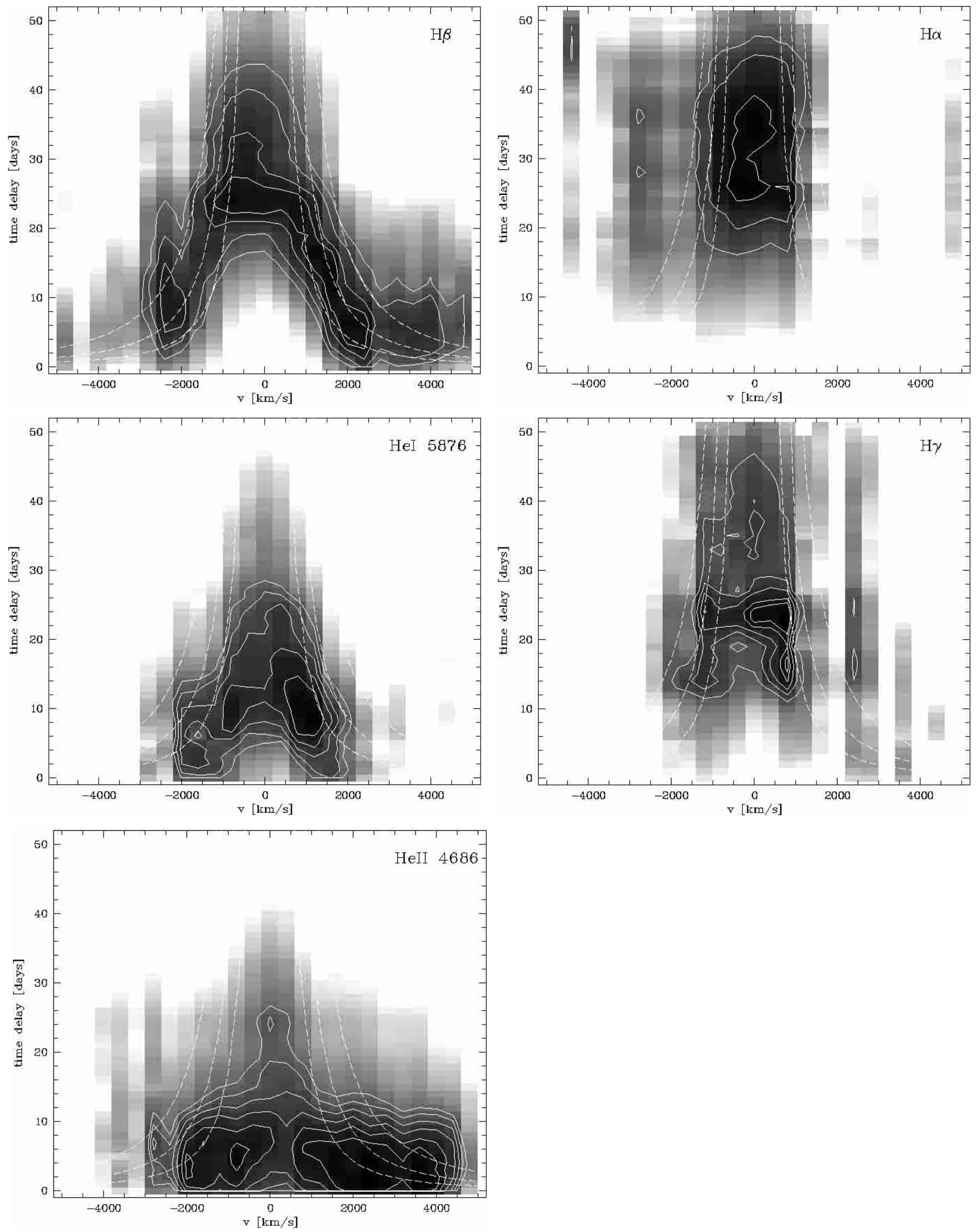

Fig. 7. The 2-D CCFs $(\tau, v)$ show the correlation of the Balmer and helium line segment light curves with the continuum light curve as a function of velocity and time delay (grey scale). Contours of the correlation coefficient are overplotted at levels between 0.800 and 0.925 (solid lines). The dashed curves show computed escape velocities for central masses of $0.5,1,2 \times 10^{7} M_{\odot}$ (from bottom to top). 


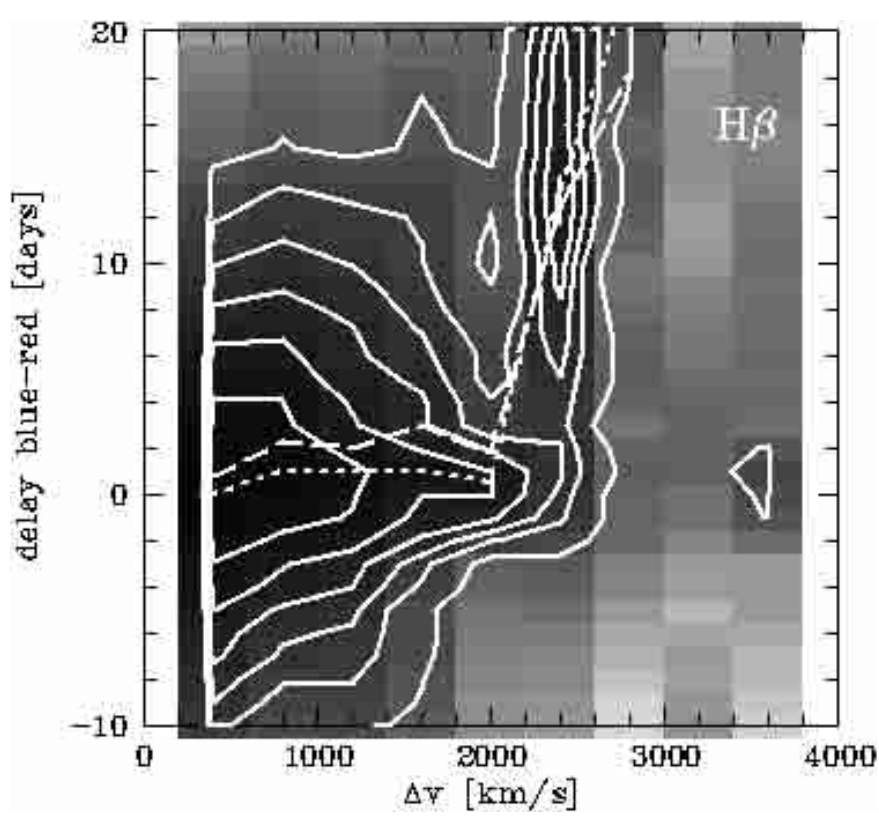

Fig. 8. Time delay $\tau$ of the blue $\mathrm{H} \beta$ line wing with respect to the red one as a function of distance to the line center. Contours of the correlation coefficient are overplotted at levels between 0.85 and 0.97 (solid lines). The short dashed line gives the center of the CCF. The long dashed line gives the centroid of the uppermost $10 \%$ of the CCF.

The 2-D $\operatorname{CCF}(\tau, v)$ is mathematically very similar to a 2-D response function $\Psi$ (Welsh 2001). In the next section the observed velocity delay maps are compared in more detail with model calculations of echo images from the BLR. The BLR Keplerian disk model of Welsh \& Horne (1991, Fig. 1c) shows remarkable coincidences with our observations. The three dashed lines overplotted additionally in Fig. 7 represent escape velocities computed for central masses of $0.5,1$, $2 \times 10^{7} M_{\odot}$ (from bottom to top).

\subsection{Delay between blue and red line wings}

As one part of our analysis we calculated cross-correlation functions $(\mathrm{CCF})$ of the blue line wings segments with respect to the red ones to determine more accurately their relative response. This was done for all the strong emission lines. We computed the relative delays in the line wings in velocity segments of $\Delta v=400 \mathrm{~km} \mathrm{~s}^{-1}$ width respectively beginning at $\pm 200 \mathrm{~km} \mathrm{~s}^{-1}$. Figures 8 to 10 show the delay of the blue line wings with respect to the red ones for the $\mathrm{H} \beta$, HeI $\lambda 5876$ and HeII $\lambda 4686$ lines (in gray scale). Contours of the correlation coefficient are overplotted at levels between 0.85 and 0.97 (solid lines). The center of the CCFs is indicated by the short dashed lines. The long dashed lines show the centroid of the uppermost $10 \%$ of the CCF.

The same trend is to be seen in all emission lines: the blue line wings show a delayed response with respect to the red ones at intermediate distances from the line center $(\Delta v=$ $500-2000 \mathrm{~km} \mathrm{~s}^{-1}$ ) by one to two days. Furthermore, there is a trend that the relative delay of the blue wings increases with distance to the line center.

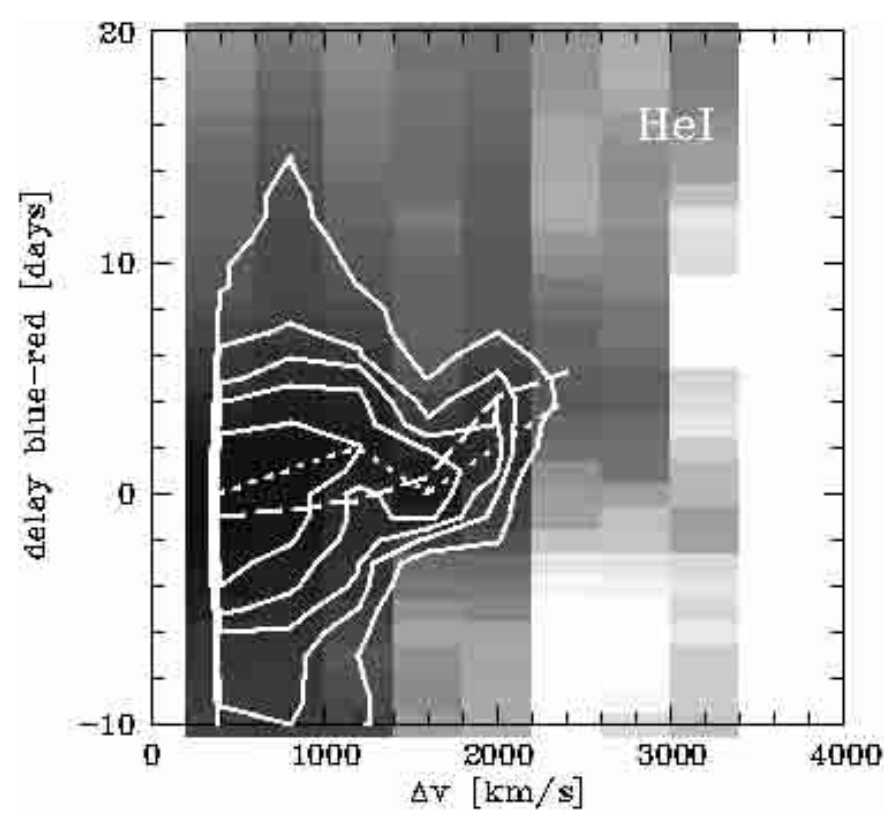

Fig. 9. Time delay $\tau$ of the blue HeI $\lambda 5876$ line wing with respect to the red one as a function of distance to the line center. Contours of the correlation coefficient are overplotted at levels between 0.85 and 0.97 (solid lines). The short dashed line gives the center of the CCF. The long dashed line gives the centroid of the uppermost $10 \%$ of the CCF.

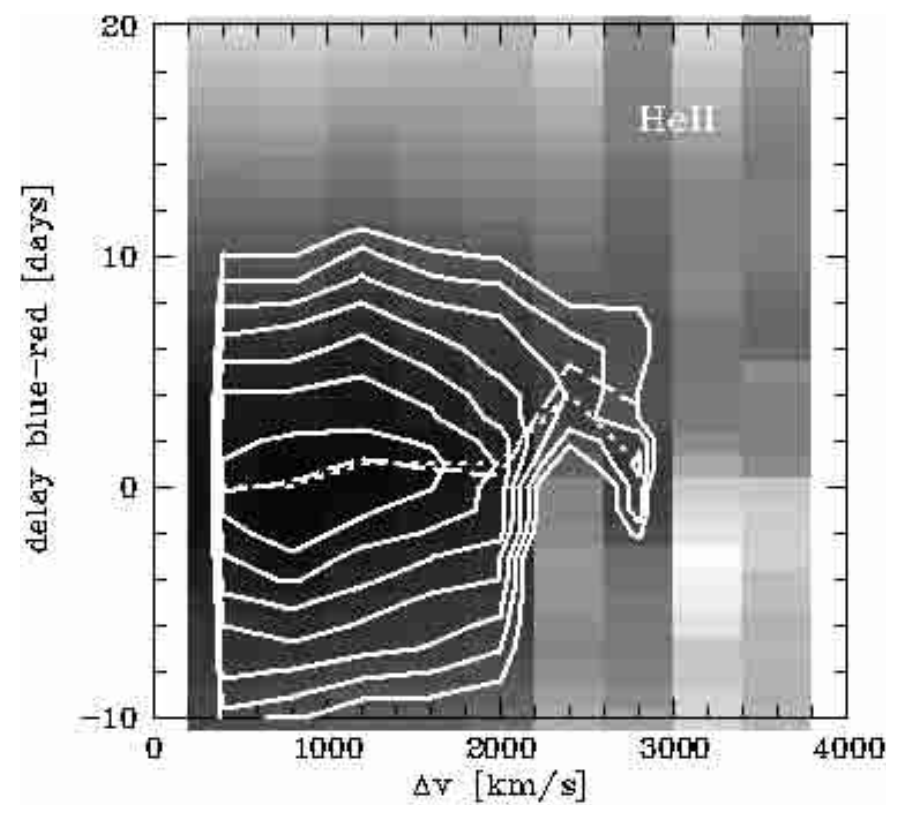

Fig. 10. Time delay $\tau$ of the blue HeII $\lambda 4686$ line wing with respect to the red one as a function of distance to the line center. Contours of the correlation coefficient are overplotted at levels between 0.85 and 0.97 (solid lines). The short dashed line gives the center of the CCF. The long dashed line gives the centroid of the uppermost $10 \%$ of the CCF.

\section{Discussion}

\subsection{BLR stratification derived from integrated lines}

The integrated emission lines in Mrk 110 respond with different delays to continuum variations as a function of ionization degree. This indicates a stratification in the broad-line region (BLR) of Mrk 110 (Kollatschny et al. 2001). Figure 2 
shows a clear correlation between time lag $\tau$ of different integrated emission lines on the one hand and their corresponding line widths $v$ on the other hand. The correlation is of the form

$\tau \propto v_{F W H M}^{-2}$

for distances $R=c \tau$ from the central ionizing source. All emission line data are consistent with a virial mass of

$M=1.8 \pm 0.3 \times 10^{7} M_{\odot}$.

The higher ionized broader emission lines originate closer to the central ionizing source than the lower ionized lines.

\subsection{BLR geometry and kinematics}

We sliced the observed emission line profiles into velocity bins of $\Delta v=400 \mathrm{~km} \mathrm{~s}^{-1}$ and correlated all the individual light curves with the continuum light curve. First results of this investigation have been published for the $\mathrm{H} \beta$ line indicating that different line segments originate at different distances from the ionizing center.

The velocity resolved profile variations of all investigated broad lines (Fig. 7) show the same characteristics. The line segments in each profile originate at different distances from the central ionizing source. The observed trend in Mrk 110 is that the outer emission line wings originate at small radii from the central supermassive black hole. This suggests strong rotational and or turbulent motions in the BLR (see however Sect. 4.3).

Comparing in more detail the observed velocity-delay pattern with BLR model calculations (Welsh \& Horne 1991; Perez et al. 1992; O'Brien et al. 1994) we can rule out that radial inflow or outflow motions - including biconical outflow - are dominant in the BLR of Mrk 110. The line wings show the shortest delay with respect to the continuum and react nearly simultaneously. Furthermore, no short delays of the central region in the Balmer and He lines are observed. This is expected in spherical BLR models with chaotic virial velocity field or randomly oriented Keplerian orbits. Therefore we can rule out a dominance of this kind of velocity field in Mrk 110. On the other hand Keplerian disk BLR models reproduce exactly the observed velocity-delay pattern i.e. the faster response of both line wings compared to the line center.

A direct comparison of the two-dimensional echo maps of the $\mathrm{H} \beta$, HeI $\lambda 5876$, and HeII $\lambda 4686$ lines with a theoretical echo image of a Keplerian disk (Welsh \& Horne 1991, Fig. 1c) is intriguing. Our observed 2D pattern of the different lines in Mrk 110 has been predicted in theoretical models which calculated the contibution from line emitting material at different radii in the BLR. The HeII $\lambda 4686$ line originates at radial distances of 3-4 light days only while $\mathrm{H} \beta$ originates at distances of about 30 light days.

A coarse estimate of the inclination angle of the accretion disk can be made by comparing the echo maps (Fig. 7) with disk models viewed at different inclination angles (Welsh \& Horne 1991, Fig. 5). We observe no short delays at the inner line regions. This excludes inclination angles of the accretion disk larger than $50^{\circ}$ in Mrk 110. At the limit of a face-on disk one expects even narrower line profiles than those we observe in Mrk 110. Therefore, a best estimate of the accretion disk inclination angle in Mrk 110 is: $30^{\circ} \pm 20^{\circ}$.

Other authors published further indications for a disk-like configuration of the broad-line region in AGN based on theoretical models or observational data (Bottorff et al. 1997; Collin-Souffrin et al. 1988; Elvis 2000; Königl \& Kartje 1994).

Velocity-delay maps have been published for the Balmer lines in NGC 5548 (Kollatschny \& Dietrich 1996) and NGC 4593 (Kollatschny \& Dietrich 1997) before. Although the quality of their spectral data was not as good as that of this campaign they could demonstrate the same basic trend as that seen in Mrk 110: the outer line wings respond faster to continuum variations than the line center. Furthermore, the red wings responded slightly faster and stronger than the blue ones in theses galaxies, too - see next section. In the UV wavelength range the variability behaviour of the prominent CIV $\lambda 1550$ line has been investigated in a few galaxies. Again there are hints for a stronger and faster response of the red wing in NGC 5548 (Chiang \& Murray 1996; Bottorff et al. 1997) and NGC 4151 (Ulrich \& Horne 1996) in comparison to the blue one. Unfortunately, the CIV $\lambda 1550$ emission lines are strongly affected by a central absorption line blend in both galaxies.

\subsection{Accretion disk wind}

In this section I will put again the main emphasis on the velocity-delay data of the $\mathrm{H} \beta$, HeII $\lambda 4686$, and $\mathrm{HeI} \lambda 5876$ lines (Figs. 7-10). A careful inspection shows that a second trend in these $2 \mathrm{D}$ velocity-delay maps is superimposed on the primary trend that both outer line wings respond faster than central line region: the response of the red line wings is stronger than that of the blue ones. For all line profiles the correlation coefficients of the red wing light curves with the continuum light curve are systematically higher by $5 \%$ (Fig. 7) than those ones of the blue wing. Furthermore, the red wings respond faster than the blue ones. The integrated blue wings (Figs. 7-10) lag the red wings by $2_{-1}^{+2}$ days.

An earlier response of the red line wing compared to the blue line wing is predicted in spherical wind and disk-wind models of the BLR (Königl \& Kartje 1994; Chiang \& Murray 1996; Blandford \& Begelman 1999). In these models the line emitting gas shows a radial outward velocity component in addition to the rotation. Also a stronger response of the red wing is expected in the models and observed in NGC 5548 (Chiang $\&$ Murray 1996). But we could not verify a secondary peak in our data as predicted in the model of Chiang \& Murray (1996). The disk outflow/wind models are distinguished from spherical wind models that their velocity decreases with radius (this means from the outer line wings to the line center) rather than the other way around. The observed delays of the blue line wings with respect to the red ones (Figs. 8-10) point at an accretion disk-wind in Mrk 110. In particular one can see an increase of the blue-red delay towards the outer line wings which means that the wind velocity increases towards the center. 


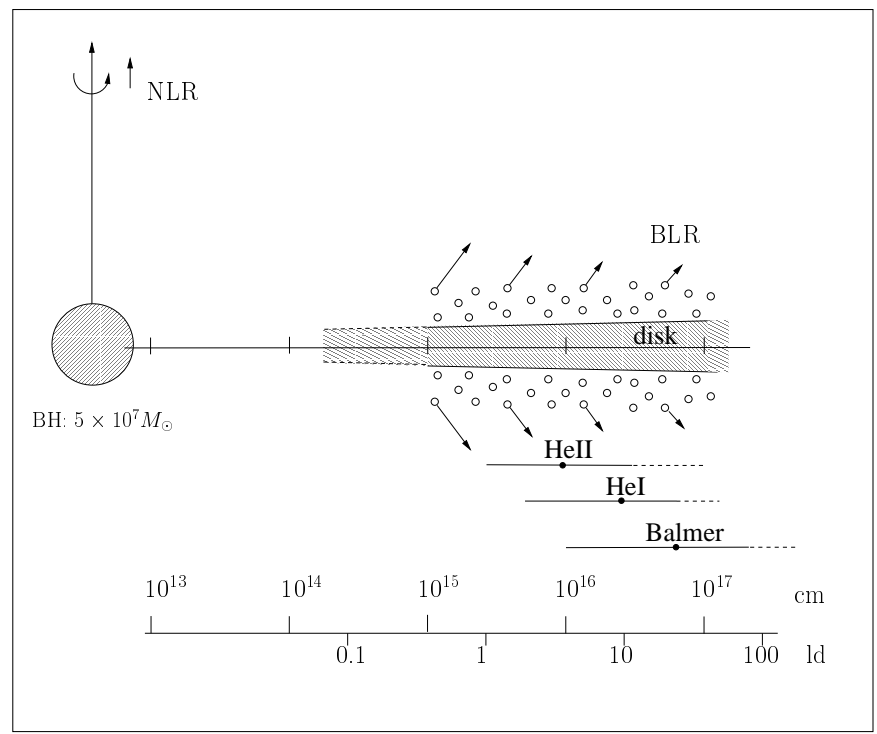

Fig. 11. Schematic BLR model of Mrk 110.

Furthermore, Murray \& Chiang (1997) demonstrated that a Keplerian disk wind reproduces single-peaked broad emission lines as we see in the spectra of Mrk 110.

\subsection{Central black hole mass}

From the integrated line intensity variations of four different emission lines a virial mass of

$M=1.8 \pm 0.3 \times 10^{7} M_{\odot}$

has been determined (Sect. 3). We made the assumption that the characteristic velocities of the particular emission line regions are given by the $F W H M$ of their rms profiles and the characteristic distances $R$ are given by the centroids of the corresponding cross-correlation functions:

$M=\frac{3}{2} v^{2} G^{-1} R$

(e.g. Koratkar \& Gaskell 1991; Kollatschny \& Dietrich 1997). Additionally, we may compare the velocity-delay maps (Fig. 7) with escape velocity envelopes

$v=\sqrt{2 G M / c \tau}$

for different central masses. With this method we derive a central black hole mass of

$M=1.5 \pm 1 \times 10^{7} M_{\odot}$

in Mrk 110. This mass value confirms perfectly our earlier derived value.

But one has to keep in mind that there are further systematic uncertainties in the mass determination (e.g. Krolik 2001). Due to the unknown inclination angle of the accretion disk the derived mass may be a lower limit only. Our observed velocitydelay maps do not drop down near the line center (Fig. 3) as is expected from model calculations for edge-on disk models (Welsh \& Horne 1991; O'Brien et al. 1994). This is a hint for a small inclination angle of the accretion disk in Mrk 110.
Ferrarese et al. (2001) measured the stellar velocity dispersion in the CaII triplet lines of the host galaxy in Mrk 110. Their derived velocity dispersion corresponds to a central mass of only

$M=3 \times 10^{6} M_{\odot}$

in their black hole mass vs. velocity dispersion diagram. This value is a factor of 5 below our black hole mass value we derived with other methods for this galaxy. But one has to consider that Mrk 110 is a galaxy system in the late stage of merging with respect to its morphology. Therefore, the derived central stellar velocity dispersion in the host galaxy of Mrk 110 might by contaminated heavily by this interaction/merging effect.

\subsection{Schematic BLR model}

We are now in the position of generating a schematic model of the innermost AGN region in Mrk 110 from all our data. The result is shown in Fig. 11. We could demonstrate that the broad emission lines originate in the wind of an accretion disk. The distances of the line emitting regions from the central ionizing source are shown on a logarithmic scale. A central black hole mass of $M=1.8 \pm 1 \times 10^{7} M_{\odot}$ is a lower limit only because of the poorly known inclination angle of the accretion disk. This value is of the order of $30^{\circ}$. Therefore, we draw a Schwarzschild radius for a black hole mass of $M=5 \times 10^{7} M_{\odot}$ in Fig. 11.

\section{Summary}

We analyzed carefully Balmer and helium emission line profile variations which we derived from our monitoring campaign on the Seyfert 1 galaxy Mrk 110. Three clear trends are to be seen in our generated velocity-delay maps:

- The outer red and blue line wings of the emission profiles respond nearly simultaneously with respect to variations of the ionizing continuum and much faster than the inner regions in the emission lines. A detailed comparison of our observed velocity-delay maps with theoretical models of other authors points clearly to an accretion disk where the broad emission lines originate.

- There is the general trend starting from the Balmer lines upon the HeI line up to the HeII lines that the higher ionized lines respond systematically faster. This is to be seen in the integrated lines on the one hand as well as in the velocity resolved line profiles on the other hand. It indicates an ionization stratification in the broad-line region.

- The response of the red line wing with respect to the ionizing continuum variations is systematically stronger and slightly faster compared to the blue line wing. This finding is the signature of an accretion disk wind in the broad-line region.

We derived from the integrated line intensity variations as well as from the line profile variations a central black hole mass of $M=1.8 \times 10^{7} M_{\odot}$. This value is a lower limit because of the unknown accretion disk orientation. 
Acknowledgements. WK thanks the UT Astronomy Department for warm hospitality during his visit. He thanks K. Bischoff, M. Bottorff, and M. Zetzl for valuable comments. Part of this work has been supported by the Deutsche Forschungsgemeinschaft, DFG grant KO 857/24 and DAAD.

\section{References}

Blandford, R. D., \& Begelman, M. C. 1999, MNRAS, 303, L1

Blandford, R. D., \& Payne, D. G. 1982, MNRAS, 199, 883

Bottorff, M., Korista, K. T., Shlosman, I., \& Blandford, R. D. 1997, ApJ, 479, 200

Chiang, J., \& Murray, N. 1996, ApJ, 466, 704

Cohen, M. H., \& Martel, A. R. 2002, in Mass outflow in AGN, ed. D. M. Crenshaw et al., ASP Conf. Ser., 255, 255

Collin-Souffrin, S., Dyson, J. E., McDowell, J. C., \& Perry, J. J. 1988, MNRAS, 232, 539

Elvis, M. 2000, ApJ, 545, 63

Emmering, R. T., Blandford, R. D., \& Shlosman, I. 1992, ApJ, 385, 460
Ferrarese, L., Pogge, R. W., Peterson, B. M., et al. 2001, ApJ, 555, L79

Königl, A., \& Kartje, J. E. 1994, ApJ, 434, 446

Kollatschny, W., Bischoff, K., Robinson, E. L., Welsh, W. F., \& Hill, G. J. 2001, A\&A, 379, 125 (Paper I)

Kollatschny, W., \& Bischoff, K. 2002, A\&A, 386, L19 (Paper II)

Kollatschny, W., \& Dietrich, M. 1996, A\&A, 314, 43

Kollatschny, W., \& Dietrich, M. 1997, A\&A, 323, 5

Koratkar, A., \& Gaskell, M. 1991, ApJ, 370, L61

Krolik, J. H. 2001, ApJ, 551, 72

Murray, N., \& Chiang, J. 1997, ApJ, 474, 91

O'Brien, P. T., Goad, M. R., \& Gondhalekar, P. M. 1994, MNRAS, 268,845

Perez, E., Robinson, A., \& de la Fuente, L. 1992, MNRAS, 256, 103

Proga, D., Stone, J. M., \& Kallman, T. R. 2000, ApJ, 543, 686

Ulrich, M.-H., \& Horne, K. 1996, MNRAS, 283, 748

Welsh, W. F., \& Horne, K. 1991, ApJ, 379, 586

Welsh, W. F. 2001, in Probing the Physics of AGN, ed. Peterson et al., ASP Conf. Ser., 224, 123 